\title{
Does the degree of preoperative mitral regurgitation predict survival or the need for mitral valve repair or replacement in patients with anomalous origin of the left coronary artery from the pulmonary artery?
}

John W. Brown, MD, ${ }^{*}$ Mark Ruzmetov, MD, PhD, ${ }^{*}$ John J. Parent, MD, Mark D. Rodefeld, MD, and Mark W. Turrentine, MD

From the Section of Cardiothoracic Surgery, Indiana University School of Medicine, Indianapolis, Ind.

Presented at the 33rd Annual Meeting of the Western Thoracic Surgical Association, June 27 to 30, 2007, Santa Ana Pueblo, New Mexico.

* Drs Brown and Ruzmetov contributed equally to this work.

Received for publication June 29, 2007; revisions received Nov 24, 2007; accepted for publication Dec 18, 2007.

Address for reprints: John W. Brown, MD, Section of Cardiothoracic Surgery, Indiana University School of Medicine, 545 Barnhill Dr, EH 215, Indianapolis, IN 46202-5123 (E-mail: jobrown@iupui.edu).

J Thorac Cardiovasc Surg 2008;136:743-8 $0022-5223 / \$ 34.00$

Copyright $\odot 2008$ by The American Association for Thoracic Surgery

doi:10.1016/j.jtcvs.2007.12.065
Background: Anomalous origin of the left coronary artery from the pulmonary artery causes severe myocardial ischemia, global left ventricular dysfunction, and annular dilatation producing varying degrees of mitral regurgitation. Mitral regurgitation secondary to the left ventricular or papillary muscle dysfunction in infants will usually improve in the absence of ongoing ischemia. The aim of this study is to determine the influence of the degree of preoperative mitral regurgitation on the early and late outcomes of patients with anomalous origin of the left coronary artery from the pulmonary artery who underwent coronary reimplantation.

Methods: Twenty-five patients underwent coronary reimplantation and 1 early patient had ligation during a 30-year period (median age, 4 months; range, 1 month to 16 years), with a median follow-up of 7 years (range, 4 months to 25 years). Before repair, 7 infants (27\%) presented in extremis requiring ventilatory and inotropic support, and 17 patients $(65 \%)$ presented with heart failure. Mitral regurgitation was present in all patients: trivial in 6 patients, mild in 12 patients, moderate in 5 patients, and severe in 3 patients. No patient underwent mitral valve repair or replacement at the time of anomalous origin of the left coronary artery from the pulmonary artery repair.

Results: Hospital survival was $92 \%$. Three patients underwent mitral valve repair or replacement at the mean time of 3.5 years (all with severe preoperative mitral regurgitation). The degree of mitral regurgitation gradually improved in all remaining patients with preoperative mild and moderate mitral regurgitation. Echocardiographic studies demonstrated improvement in left ventricular function in all children. None of the patients showed any evidence of supravalvar pulmonary stenosis as a result of their pulmonary artery reconstruction.

Conclusion: Long-term clinical outcome and left ventricular function are good despite severe left ventricular dysfunction at presentation. Mitral valve repair or replacement is generally not necessary at the time of anomalous origin of the left coronary artery from the pulmonary artery repair. Significant residual mitral regurgitation is present in some patients and can usually be managed surgically at a later date depending on its degree of severity.

$\Lambda$ nomalous origin of the left coronary artery from the pulmonary artery (ALCAPA) is a rare congenital anomaly that often insidiously presents with infarction in early infancy. First described in 1885, the clinical syndrome was outlined by Bland and colleagues 1933. ${ }^{1}$ They demonstrated the combined effects of the absence of a normal coronary flow with a coronary steal and the profound ischemia that can produce left ventricular (LV) dysfunction and mitral regurgitation (MR).

Several surgical procedures have been described, initially palliative, but more recently restoring a 2-coronary system, including various forms of bypass graft 


\section{Abbreviations and Acronyms \\ ALCAPA $=$ anomalous origin of the left coronary artery from the pulmonary artery \\ ECMO = extracorporeal membrane oxygenation \\ LCA $=$ left coronary artery \\ $\mathrm{LV} \quad=$ left ventricular \\ MR = mitral regurgitation \\ MVR = mitral valve replacement \\ PA $=$ pulmonary artery}

from the aorta, ${ }^{2,3}$ subclavian artery anastomosis, ${ }^{4}$ intrapulmonary bypass graft, ${ }^{5}$ intrapulmonary artery baffle, ${ }^{6}$ and direct coronary translocation to the aorta. ${ }^{7-9}$ What remains controversial is whether to surgically treat MR at the time of ALCAPA repair. In ALCAPA, even severe MR has been reported to fully regress after reimplantation of the left coronary artery (LCA) into the aorta. ${ }^{3,8}$

We reviewed our 30-year experience with the surgical treatment of ALCAPA in infants and children. All patients but 1 were treated with the establishment of a 2-coronary system by directly reimplanting the anomalous LCA into the aorta and, in general, not treating the mitral valve surgically at the time of ALCAPA repair.

\section{Materials and Methods Study Patients}

During a 30-year period (from 1975), 26 patients aged 1 month to 16 years (mean, $33.6 \pm 63.3$ months; median, 4 months) underwent operations at the James Whitcomb Riley Hospital for Children, Indianapolis, Indiana. Thirteen patients $(50 \%)$ were female, and 13 patients $(50 \%)$ were male. Eighteen patients $(69 \%)$ were aged less than 6 months at the time of presentation and repair.

Clinical examination, chest roentgenograms, electrocardiogram, and echocardiography were performed preoperatively in most patients. At clinical presentation, 7 patients (27\%) were critically ill (requiring mechanical ventilatory and inotropic support), 3 patients $(12 \%)$ presented after an episode of ventricular fibrillation, 17 patients $(65 \%)$ presented with congestive heart failure, 6 patients (23\%) presented with a history of fatigue, 3 patients (12\%) presented with angina, and 2 patients $(8 \%)$ were asymptomatic but had a murmur. The duration of symptoms before surgical repair ranged from 2 days to 15 months. Electrocardiographic evidence of a transmural myocardial infarction, defined as the presence of Q waves in leads I and AVL, was present in 6 patients (23\%). Preoperative shortening fraction was calculated in recent patients by 2-dimensional echocardiography using both parasternal long- and short-axis views.

The median preoperative ejection fraction was $20 \%$ (mean, $23.1 \% \pm 11.9 \%$; range $10 \%-54 \%$ ). Fourteen patients $(54 \%)$ had a preoperative ejection fraction less than $20 \%$. The mean preoperative $\mathrm{LV}$ end-diastolic diameter was $3.9 \pm 0.9 \mathrm{~cm}$. When indexed to the 95th percentile of normal, the mean preoperative LV enddiastolic diameter was $1.4 \pm 0.4 \mathrm{~cm}$ (range, $0.8-2.4$ ). Preoperative mitral valve regurgitation, evaluated by 2 -dimensional echocardiography and color Doppler flow in most patients, was graded as trivial in 6 patients, mild in 12 patients, moderate in 5 patients, and severe in 3 patients. Moderate to severe preoperative mitral valve regurgitation (grade 2 to $4+$ ) was detected in 8 patients $(31 \%)$.

The indication for surgery was the diagnosis of ALCAPA. A definitive diagnosis was made by echocardiography alone with demonstration of diastolic color Doppler flow from the anomalous coronary artery into the pulmonary artery (PA) in 8 patients (31\%). The echocardiographic suspicion of ALCAPA was confirmed by cardiac catheterization in the remaining 18 patients $(69 \%)$.

\section{Surgical Technique}

Operations for all patients were performed via a median sternotomy with bicaval cannulation and hypothermic cardiopulmonary bypass. Cardiac arrest was induced by infusion of cold blood potassium cardioplegic solution through the aortic root, with crossclamping of both great vessels. The preferred method of handling the anomalous coronary artery was its removal from the transected main PA and direct reimplantation into the aorta after generous coronary mobilization. The aorta was incised laterally to assist in identifying an appropriate area above in the left sinus of Valsalva and to avoid damage or distortion of the aortic valve. This area was generally slightly higher than the usual origin of the left main coronary artery. Relocation of the coronary button was accomplished under direct vision via an adequate opening in the lateral aortic wall to avoid any damage to the aortic valve leaflets or commissures. Whenever possible, the coronary button was relocated above the posterior aortic sinus. The posterior defect in the PA was patched with autologous pericardium in all patients, and the PA was closed. The mean time of cardioplegic cardiac arrest was $44 \pm 12$ minutes (range, 19-137 minutes). The mean cardiopulmonary bypass time was $102 \pm 28$ minutes (range, 40-284 minutes).

In addition to reimplantation of the anomalous LCA into the aorta, other procedures were performed in 8 patients, including closure of the atrial septal defect $(n=3)$, ligation of a patent ductus arteriosus $(n=2)$, end-to-end method of coarctation repair $(n=1)$, and a partial anomalous pulmonary vein return repair $(n=2)$. Sternal closure was delayed in 7 patients.

An extracorporeal membrane oxygenation (ECMO) circuit was used for mechanical circulatory support in 4 patients and interaortic balloon counterpulsation was used in 1 patient early in our experience, in whom multiple attempts to wean from cardiopulmonary bypass failed after prolonged reperfusion. Right and occasionally left atrial venous and ascending aortic cannulation were used in all patients.

\section{Statistical Analysis}

Data are described as frequencies, medians with ranges, and means with standard deviations. With missing data, the number of nonmissing values is given. The characteristics and outcomes of patients in the preoperative period versus those at last follow-up were compared using Fisher exact tests, chi-square tests, $t$ tests, and multivariate logistic regression analysis of variance as appropriate. Estimates of time-related survival and freedom from reoperation after reimplantation were calculated using the Kaplan-Meier method.

Factors associated with postimplantation mortality, need for LV support, time to extubation from mechanical ventilation, discharge from the intensive care unit, and development of postoperative ventricular arrhythmias were sought in Cox's proportionate hazard 
modeling or stepwise multiple logistic regression, with patients censored at the time of death as appropriate. The variables assessed as possible predictors of these outcomes included age, sex, weight, body surface area, preoperative symptom severity and duration, initial presentation with ventricular arrhythmia, preoperative infarction or ischemia by electrocardiography, preoperative ejection fraction, $\mathrm{LV}$ end-diastolic diameter, severity of MR, presence of associated diagnoses, date of ALCAPA repair by aortic reimplantation, and aortic crossclamp time. The need for postoperative ECMO or an aortic balloon pump was also used as a variable assessed as a predictor of late outcomes.

Differences in survival curves were assessed by log-rank test. All analyses were performed with the Statistical Package for the Social Sciences (SPSS Inc, Chicago, Ill). Early mortality was defined as death during initial hospitalization or within 30 days of operation. Any deaths later than that were defined as late mortality. In this series, all early deaths occurred during the initial hospitalization and all late deaths occurred after discharge from the initial hospitalization.

\section{Results}

\section{Mortality}

There were 2 early deaths and 1 late death, accounting for an overall mortality of $12 \%$. No mortality has occurred since 1995. The cause of the first early death was sustained ventricular arrhythmias on postoperative day 12; the patient did not respond to medical therapy and multiple defibrillation attempts. The second patient underwent ALCAPA repair with an ejection fraction of $10 \%$. The patient required ECMO after the procedure and stayed on ECMO for 15 days. On day 8 , cerebral hemorrhage was detected and was progressive on serial head ultrasound examinations. The patient was weaned from ECMO and was transferred to a community hospital, where he died 1 month after surgery. This patient died 29 days after surgery from his neurologic injury. He had been weaned from ECMO and his cardiac status was improving.

The late death occurred in a patient who initially underwent ALCAPA ligation in 1975 as an infant. Preoperatively, this patient had severe MR, but at the time of ALCAPA ligation the mitral valve was left intact. Three years later, this patient underwent mitral valve replacement (MVR) with mechanical prosthesis. The mechanical valve was replaced twice at 15 and 17 years after the initial MVR. The patient's ALCAPA was bypassed with the left internal thoracic artery 16 years after ALCAPA ligation. This patient died 25 years after the initial ALCAPA ligation because of bacterial endocarditis on the prosthetic valve.

Kaplan-Meier survival is $88 \% \pm 6 \%$ at 25 years (Figure 1). Univariate logistic regression identified the time of surgery (before 1995) as a potential risk factor of mortality $(P=.02)$. Logistic regression demonstrates that severe MR was not correlated with a higher probability of death. Multivariate logistic regression modeling indicates no independent predictors of death.

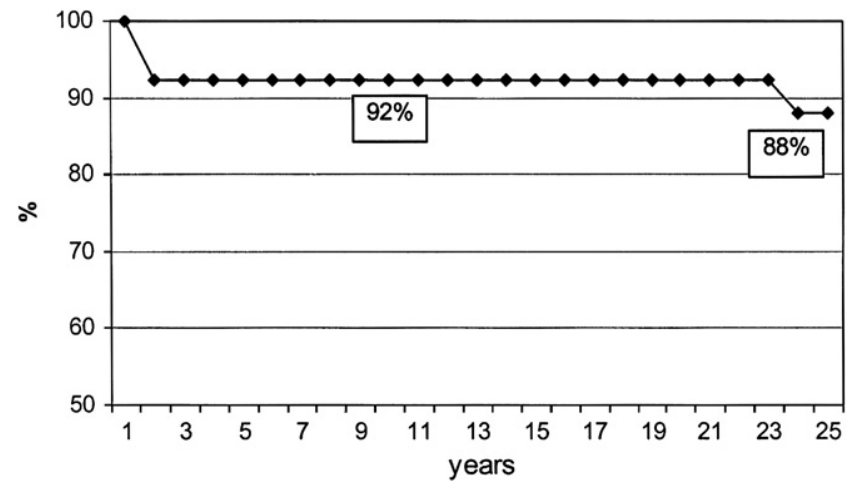

Figure 1. Kaplan-Meier estimated 25-year survival, including hospital mortality.

\section{Reoperation}

Four patients underwent reoperation after ALCAPA repair: Three patients had severe MR (MVR in 2 patients, and mitral valve repair in 1 patient), and 1 patient had patch arterioplasty of a stenotic left main coronary artery. One patient (age, 3 years) underwent MVR (size $25 \mathrm{~mm}$; St Jude Medical, Inc, Minneapolis, Minn) 2 years after ALCAPA repair. One patient (age, 5 months) had a DeVega (annuloplasty stitch) mitral valve annuloplasty 3.5 years after the initial ALCAPA repair. The third patient (age, 6 months) underwent MVR 3 years after ALCAPA ligation (size 26 mm; Beall-Surgitool, Medical Engineering Corp, Racine, Wis), but then underwent repeat MVR twice 9 and 11 years after the initial MVR (size number 3, Starr-Edwards, Edwards Laboratories, Irvine, Calif; size $31 \mathrm{~mm}$, St Jude Medical, Inc) and died of mitral prosthetic endocarditis 25 years after ALCAPA surgery. The fourth patient with left main coronary artery stenosis underwent left main patch augmentation 9 years after the initial surgery. No patient underwent a repeat PA repair. Freedom from reoperation at 25 years was $83 \% \pm 9 \%$ (Figure 2 ). Univariate and multivariate logistic regression identified preoperative severe MR as a risk factor for reoperation $(P<.001)$.

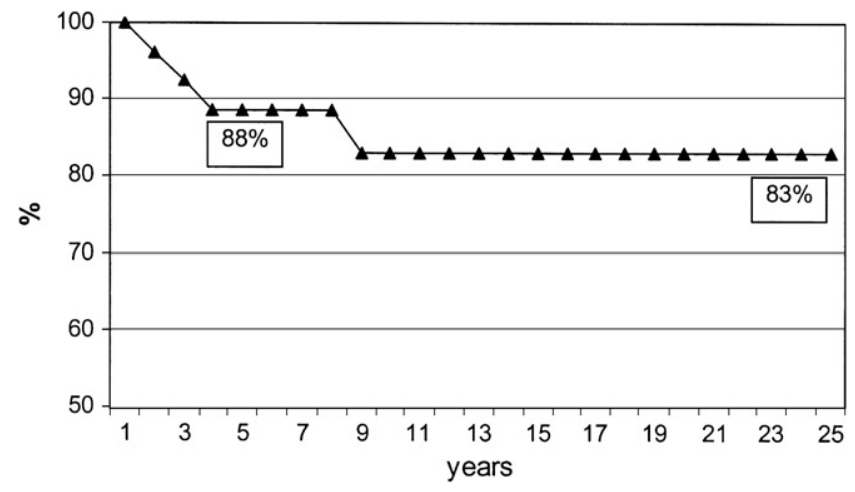

Figure 2. Kaplan-Meier estimated 25-year freedom from reoperation. 


\section{Late Echocardiographic Follow-up}

Follow-up echocardiography $(n=24)$ at a mean interval of $10.1 \pm 8.2$ years (median, 7 years; range, 4 months to 25 years) demonstrated a mean ejection fraction of $56.0 \% \pm$ $13.2 \%$ (compared with a mean preoperative ejection fraction of $23.1 \% \pm 11.9 \% ; P<.0001)$. MR was trivial in 12 patients, mild in 10 patients, moderate in 2 patients, and severe in 1 patient (all 3 patients with moderate or greater MR have undergone MVR or repair). The degree of MR improved significantly during the follow-up period (moderate or severe MR $31 \%$ preoperatively compared with $4 \%$ at last followup; $P=.02$ ). Residual wall motion abnormalities were found in 16 patients and absent in 8 patients, with persistent septal hypokinesis in 2 patients $(8 \%)$. Preoperative wall motion abnormalities were observed in $81 \%$ of patients $(P=.02)$. At follow-up in 18 patients, the ratio of the measured LV end-diastolic diameter to the 95th percentile of normal at the follow-up period had decreased from $1.6 \pm 0.3$ to 1.0 \pm 0.3 (range, $0.8-1.4 ; P<.0001$ ). All patients showed significant improvement after coronary reimplantation. Color Doppler echocardiograms in 22 patients indicated that the reimplanted LCA was widely patent in all patients during the follow-up period except for the 1 patient who developed left main stenosis and subsequently had a patch angioplasty.

Normalization of ejection fraction, resolution of LV dilatation, improvement in MR, and wall motion abnormalities usually occurred within 1 year of repair (Figure 3). By mixed linear regression analysis, the ejection fraction normalized by 5 months, LV dilatation normalized by 6 months, and degree of MR improved to mild or less by 9 months after the initial repair except in the 3 patients who eventually required MVR or repair.

\section{Discussion}

ALCAPA is a rare congenital anomaly that often causes myocardial ischemia or infarction within the first few weeks or months of life, leading to LV dysfunction, mitral valve incompetence, and congestive heart failure. Several surgical methods have been developed for treating ALCAPA. Ligation of the LCA at its origin from the PA is rarely performed today because of high early and late mortality, especially in small infants. ${ }^{10,11}$ Establishing a 2-coronary system has been the main goal of surgical therapy for the last 3 decades. The continuity of the LCA with the aorta may be established by several methods. In 1966, Cooley and colleagues ${ }^{2}$ reported 2 cases of ALCAPA in children in whom an LCA-aortic anastomosis was created with a Dacron graft in 1 case and an autologous saphenous vein in 1 case. Some surgeons recommend anastomosis with the left or right subclavian artery to preclude the necessity of cardioplegic arrest. ${ }^{12}$ Anastomosis between the internal thoracic artery and the LCA has also been proposed as a surgical method for treating ALCAPA, ${ }^{13}$ but this procedure is difficult to perform in small infants and the late potency of the anastomosis

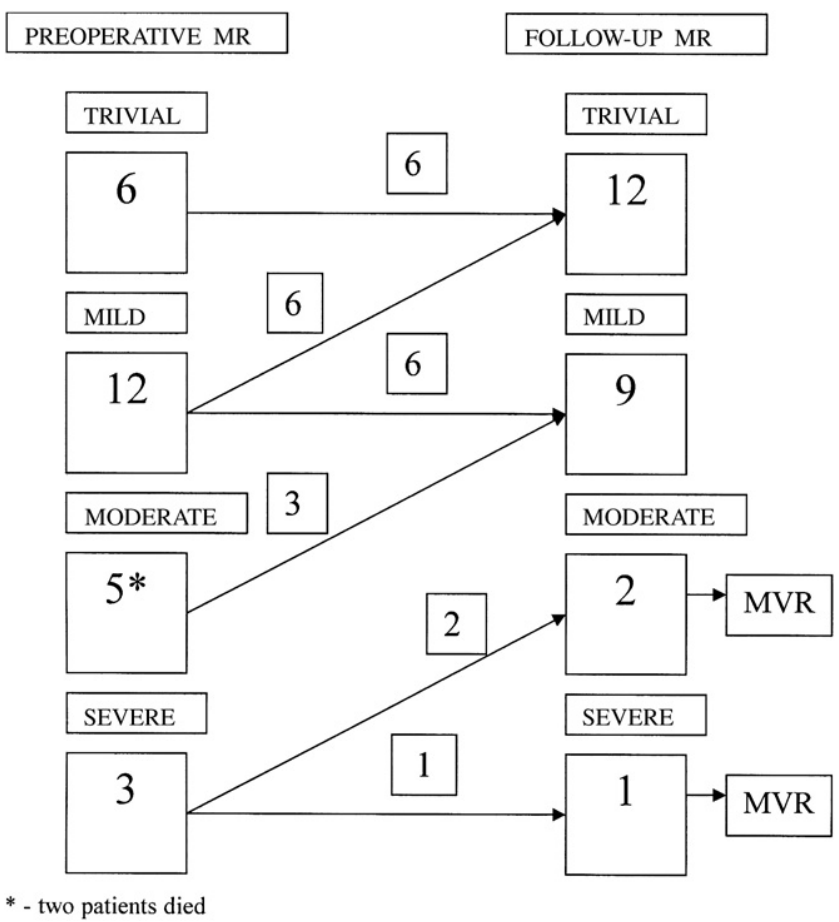

Figure 3. Longitudinal changes in the degree of MR with the corresponding number of patients at risk. MVR, Mitral valve replacement; $M R$, mitral regurgitation.

is uncertain. A 2-coronary system can be created by a Takeuchi operation. ${ }^{6}$ The mortality associated with this procedure is low, but occurrences of supravalvular pulmonary stenosis, baffle obstruction, and aortic regurgitation in the late postoperative period have been reported. ${ }^{14-16}$ Direct reimplantation of the LCA to the aorta is an attractive approach. First reported by Neches and colleagues, ${ }^{7}$ this procedure has been successfully used in infants and adults. The operation is coupled with a low mortality and a high rate of anastomotic patency, and it usually brings about a dramatic improvement in LV function, ${ }^{15,17}$ as substantiated by the results of this and other studies.

The severity of preoperative MR has also been identified as a risk factor for death after surgery, ${ }^{18}$ although this is not supported unanimously. ${ }^{17,19,20}$ Review of our experience did not identify severe MR as a risk factor for mortality. Both early deaths in this series occurred in infants with mild to moderate regurgitation. On the basis of our experience, we have developed a general policy not to address MR by repair or replacement at the initial operation. In ALCAPA, even severe MR has been reported to fully regress after reperfusion alone in the majority of cases. ${ }^{21,22}$ In the present study, mitral valve regurgitation had already improved significantly by the time of hospital discharge when compared with the preoperative findings. Furthermore, freedom from MR greater than mild at 25 years was $88 \%$ in all but the 3 patients who had 
undergone MVR or repair. Thus, the primary goal of the initial surgery should be to establish antegrade coronary perfusion.

Some authors ${ }^{23}$ recommend routine mitral valve repair at the time of coronary artery revascularization on the grounds that early postoperative cardiac output is improved and operative mortality is reduced. It remains speculative whether their excellent results would have been achieved without associated mitral valve procedures or if their patients were mostly infants, as in our study. Others advocate mitral annuloplasty $^{15,20,24}$ or MVR ${ }^{14,24}$ only in the presence of severe MR. In our mostly infant patient population, the mitral valve was not addressed at the initial repair. When we analyzed our results and adverse outcomes, our philosophy of not repairing moderate or severe MR at the initial ALCAPA repair supports our approach. All 3 patients with severe preoperative MR also had severe LV dysfunction. All 3 infants survived ALCAPA repairs and in our opinion would have been better candidates for a definitive mitral valve repair $(n=1)$ or replacement $(n=2)$ if they had been older, they had recovered from the myocardial ischemia caused by ALCAPA, and their left ventricles had improved or returned to normal function. The 2 early deaths in the 5 patients who presented with moderate MR were infants ( $<4$ months old) with severe LV dysfunction $(\mathrm{EF}<20 \%)$. One patient died of an arrhythmia 12 days after coronary reimplantation when LV function was beginning to improve. The other infant died of a cerebral bleed as he was being weaned successfully from ECMO. He died 3 weeks later of neurologic complications, but his cardiac function had already improved significantly. We doubt that mitral valve repair would have changed these patients' outcomes. We would agree with Isomatsu and coauthors ${ }^{23}$ that mitral valve repair can and should be considered in an older child if the regurgitation is moderately severe or severe and they cannot be weaned from mechanical support.

MVR or repair during ALCAPA repair would prolong the ischemic insult to the sick infant ventricle and is potentially harmful by acutely increasing the afterload in an already damaged ventricle. Severe MR was a risk factor for reoperation but not a risk factor for death in this series.

Occlusion or stenosis of the LCA after reimplantation of the ALCAPA is rarely seen. Stenosis or occlusion is usually related to the excessive tension placed on this vessel to reach the aorta and from its distant position on the posterolateral aspect of the main PA. Disruption of the vasa vasorum may also play a role. When late obstruction of the left coronary occurs after the reimplantation, revascularization of the LCA can be accomplished by patch angioplasty of the left main coronary artery $^{25}$ or coronary bypass using an internal thoracic artery. Coronary bypass with the left internal thoracic artery can be successfully performed even in very young children. ${ }^{26}$

Encouraging results have been achieved with the use of cardiac mechanical support as a bridge to recovery in the postoperative setting of ALCAPA. ${ }^{15,21,25,27,28}$ Such support measures include LV assist devices, ${ }^{21,27}$ ECMO, ${ }^{15,25,29}$ and intra-aortic balloon pumps ${ }^{30}$ in older children. In a report by Del Nido and associates, ${ }^{27} 5$ survivors among 7 infants who underwent surgical correction of ALCAPA and intractable LV failure were successfully supported with an LV assist device. In our series of patients with ALCAPA, we have not used LV assist devices, but 5 infants required short-term postoperative mechanical support after direct aortic reimplantation and 4 infants were long-term survivors. Mechanical assistance improves results and maximally enhances patient survival; LV assist devices and ECMO play an obvious integral part in the modern surgical treatment of patients with ALCAPA.

\section{Conclusions}

ALCAPA repair by establishing a 2-coronary myocardial perfusion offers the best potential for $\mathrm{LV}$ functional recovery. Coronary reimplantation offers superior long-term survival and a higher freedom from reoperation than alternative techniques. MVR or repair is generally not necessary at the time of the initial operation and is potentially harmful by acutely increasing LV afterload in these sick ventricles. All 3 patients in this series who presented with severe MR tolerated a 2+year delay for their subsequent MVR or repair. Moderate MR will generally improve in the majority of patients after ALCAPA repair, and MV regurgitation should only be corrected in the rare patient who cannot be weaned from bypass or postoperative mechanical support.

\section{References}

1. Bland EF, White PD, Garland J. Congenital anomalies of the coronary arteries. Am Heart J. 1933;8:787-801.

2. Cooley DA, Hallman GL, Bloodwell RD. Definitive surgical treatment of anomalous origin of left coronary artery from pulmonary artery: indications and results. J Thorac Cardiovasc Surg. 1966;52:798-808.

3. Chaitman BR, Bourassa MG, Lesperance J, Grondin P. Anomalous left coronary artery from pulmonary artery: an eight year angiographic follow-up after saphenous vein bypass graft. Circulation. 1975;51: 552-5.

4. Meyer BW, Stefanik G, Stiles QR, Lindesmith GG, Jones JC. A method of definitive surgical treatment of anomalous origin of left coronary artery. J Thorac Cardiovasc Surg. 1968;56:104-7.

5. Arciniegas RR, Farooki ZQ, Hakimi M, Green EW. Management of anomalous left coronary artery from the pulmonary artery. Circulation. 1980;62:I180-9.

6. Takeuchi S, Imamura H. Katsumoto, et al. New surgical method for repair of anomalous left coronary artery from pulmonary artery. $J$ Thorac Cardiovasc Surg. 1979;78:7-11.

7. Neches WH, Mathews RA, Park SC, et al. Anomalous origin of the left coronary artery from the pulmonary artery. Circulation. 1974;50:582-7.

8. Grace RR, Angelini P, Cooley DA. Aortic implantation of anomalous left coronary artery arising from pulmonary artery. Am J Cardiol. 1977;39:608-13.

9. Laborde F, Marchand M, Leca F, Jarreau M, Dequirot A, Hazan E. Surgical treatment of anomalous origin of the left coronary artery in infancy and childhood. J Thorac Cardiovasc Surg. 1981;82:423-8.

10. Kececioglu D, Voth E, Morguet A, Munz DL, Vogt J. Myocardial ischemia and left-ventricular function after ligation of left coronary artery (Bland-White-Garland syndrome): a long-term follow-up. Thorac Cardiovasc Surg. 1992;40:283-7. 
11. Wilson CL, Dlabal PW, McGuire SA. Surgical treatment of anomalous left coronary artery from pulmonary artery: follow-up in teenagers and adults. Am Heart J. 1978;98:440-6.

12. Kesler KA, Pennington DG, Nouri S, et al. Left subclavian-left coronary artery: long-term follow-up. J Thorac Cardiovasc Surg. 1989;98:25-9.

13. Kitamura S, Kawachi K, Nishii T, et al. Internal thoracic artery grafting for congenital coronary malformations. Ann Thorac Surg. 1992;53:513-6.

14. Bunton R, Jonas RA, Lang P, Rein AJ, Castaneda AR. Anomalous origin of left coronary artery from pulmonary artery. Ligation versus establishment of a two-coronary system. J Thorac Cardiovasc Surg. 1987;93: 103-8.

15. Alexi-Meskishvili V, Hetzer R, Weng Y, Loebe M, Lange PE, Ishino K. Successful extracorporeal circulatory support after aortic reimplantation of anomalous left coronary artery. Eur J Cardiothorac Surg. 1994;8: 533-6.

16. Tkebuchava T, Carrel T, van Segesser L, Real F, Jenni R, Turina M. Repair of anomalous origin of the left coronary artery from the pulmonary artery without early and late mortality in 9 patients. J Cardiovasc Surg (Torino). 1992;33. 479-475.

17. Vouhé PR, Tamisier D, Sidi D, et al. Anomalous left coronary artery from the pulmonary artery: results of isolated aortic reimplantation. Ann Thorac Surg. 1992;54:621-7.

18. Schwartz ML, Jonas RA, Colan D. Anomalous origin of left coronary artery from pulmonary artery: recovery of left ventricular function after dual coronary repair. J Am Coll Cardiol. 1997;30:547-53.

19. Sauer U, Stern H, Meisner H, Buhlmeyer K, Sebening F. Risk factors for perioperative mortality in children with anomalous origin of the left coronary artery from the pulmonary artery. $J$ Thorac Cardiovasc Surg. 1992;104:696-705.

20. Lambert V, Touchot A, Losay J, et al. Midterm results after surgical repair of the anomalous origin of the coronary artery. Circulation. 1996; 94:1138-43.

21. Cochrane AD, Coleman DM, Davis AM, Brizard CP, Wolfe R, Karl TR. Excellent long-term functional outcome after an operation for anoma- lous left coronary artery from the pulmonary artery. $J$ Thorac Cardiovasc Surg. 1999;117:332-42.

22. Jin Z, Berger F, Uhlemann F, et al. Improvement in left ventricular dysfunction after aortic reimplantation in 11 consecutive paediatric patients with anomalous origin of the left coronary artery from the pulmonary artery. Early results of a serial echocardiographic follow-up. Eur Heart J. 1994; $15: 1044-9$

23. Isomatsu Y, Imai Y, Shin'oka T, Aoki M, Iwata Y. Surgical intervention for anomalous origin of the left coronary artery from the pulmonary artery: the Tokyo experience. J Thorac Cardiovasc Surg. 2001;121: 792-7.

24. Yam MC, Menahem S. Mitral valve replacement for severe mitral valve regurgitation in infants with anomalous left coronary artery from the pulmonary artery. Pediatr Cardiol. 1996;17:271-4.

25. Huddleston CB, Balzer DT, Mendeloff EN. Repair of anomalous left main coronary artery arising from the pulmonary artery in infants: long-term impact on the mitral valve. Ann Thorac Surg. 2001;71: 1985-9.

26. Mavroudis C, Backer CL, Muster AJ, et al. Expanding indications for pediatric coronary bypass. J Thorac Cardiovasc Surg. 1996;111:181-9.

27. Del Nido PJ, Duncan BW, Mayer JE, Wessel DL, LaPierre RA, Jonas RA. Left ventricular assist device improves survival in children with left ventricular dysfunction after repair of anomalous origin of the left coronary artery from the pulmonary artery. Ann Thorac Surg. 1999;67:169-72.

28. Dodge-Khatami A, Mavroudis C, Backer CL. Anomalous origin of the left coronary artery from the pulmonary artery: collective review of surgical therapy. Ann Thorac Surg. 2002;74:946-55.

29. Delius RE, Caldarone C. Mechanical support of the pediatric cardiac patient. Semin Thorac Cardiovasc Surg Pediatr Cardiac Surg Annu. 2000;3179-85.

30. Pozzi M, Santoro G, Makundan S. Intraaortic balloon pump after treatment of anomalous origin of left coronary artery. Ann Thorac Surg. 1998;65:555-7. 